\title{
ARTICLE OPEN \\ The origin of electronic band structure anomaly in topological crystalline insulator group-IV tellurides
}

\author{
Zhen-Yu Ye ${ }^{1,2}$, Hui-Xiong Deng ${ }^{2}$, Hui-Zhen $\mathrm{Wu}^{1}$, Shu-Shen $\mathrm{Li}^{2}$, Su-Huai Wei ${ }^{3}$ and Jun-Wei Luo ${ }^{2}$
}

Group-IV tellurides have exhibited exotic band structures. Specifically, despite the fact that Sn sits between Ge and Pb in the same column of the periodic table, cubic SnTe is a topological crystalline insulator with band inversion, but both isovalent GeTe and PbTe are trivial semiconductors with normal band order. By performing first-principles band structure calculations, we unravel the origin of this abnormal behaviour by using symmetry analysis and the atomic orbital energy levels and atomic sizes of these elements. In group-IV tellurides, the $s$ lone pair band of the group-IV element is allowed by symmetry to couple with the anion valence $p$ band at the $L$-point, and such s-p coupling leads to the occurrence of bandgap at the $L$-point. We find that such s-p coupling is so strong in $\mathrm{SnTe}$ that it inverts the band order near the bandgap; however, it is not strong enough in both GeTe and PbTe, so they remain normal semiconductors. The reason for this is the incomplete screening of the core of the relatively tight-binding Ge $4 \mathrm{~s}$ orbital by its $3 \mathrm{~d}$ orbitals and the large atomic size and strong relativistic effect in $\mathrm{Pb}$, respectively. Interestingly, we also find that the rhombohedral distortion removes the inversion symmetry and the reduced s-p coupling transforms the $a$-SnTe back to a normal semiconductor. Our study demonstrates that, in addition to spin-orbital coupling, strain and interface dipole fields, inter-orbital coupling is another effective way to engineer the topological insulators.

npj Computational Materials (2015) 1, 15001; doi:10.1038/npjcompumats.2015.1; published online 25 November 2015

\section{INTRODUCTION}

Topological insulators (TIs) are a group of materials with interesting electronic properties. They can exhibit insulating state in the bulk, but support spin-momentum locked gapless states at the boundaries. For three-dimensional $\mathrm{Tls}^{1,2}$ the metallic topological surface states induced by spin-orbit coupling (SOC) are protected by time-reversal symmetry, known as Kramer's pair. Time-reversal symmetry forbids the elastic backscattering of Kramer's pair surface states, and the dispassion-less transport of the topological surface states is robust against nonmagnetic weak disorder. Topological crystalline insulators ${ }^{3}$ (TCls) are new topological phases of matter in which the topological surface states are protected by crystal symmetries, ${ }^{4}$ such as the mirror symmetry of the crystal with respect to $\{110\}$ plane in the rocksalt (RS) structure of semiconductor SnTe, instead of time-reversal symmetry in Tls. The SnTe-like TCl state is characterised by a new topological invariant called the mirror Chern number, instead of the $Z_{2}$ invariant in general Tls.

The RS structure IV-VI narrow band gap semiconductors such as GeTe, SnTe and PbTe and their alloys have been extensively explored in developing infrared optoelectronic devices ${ }^{5-8}$ and as high-performance thermoelectric materials. ${ }^{9}$ Besides their wide range of applications, the IV-VI materials also exhibit exotic electronic structures. For example, SnTe was recently found to be the prototype of TCls. The topological surface states of SnTe and related IV-VI group alloys $\mathrm{Pb}_{1-x} \mathrm{Sn}_{x} \mathrm{Te}$ and $\mathrm{Pb}_{1-x} \mathrm{Sn}_{x} \mathrm{Se}$ have been theoretically predicted by performing the first-principles calculations $^{4}$ and experimentally observed by carrying out angle-resolved photoemission spectroscopy, ${ }^{10-13}$ scanning tunnelling microscope, ${ }^{14,15}$ as well as transport measurements. ${ }^{16,17}$ Surprisingly, although SnTe is a well-known TCl, both of its lighter isovalent GeTe and heavier isovalent PbTe counterparts are normal insulators. This non-monotonic behaviour of IV-Te compounds is quite unexpected and the origin has not been fully explained.

In this paper, performing first-principles calculations, we unravel the origin of the anomalies by studying the electronic structures of GeTe, SnTe and PbTe. We find that in the cubic (RS, $\beta$-phase) the (low lying) cation $s$ lone-pair state, which are valence electrons that are not shared with another atom, couples strongly with (high lying) anion valence $p$ state that leads to band inversion in SnTe, but the coupling is not large enough for GeTe due to its low $4 \mathrm{~s}$ orbital energy and for $\mathrm{PbTe}$ due to its large $\mathrm{Pb}$ atom size and relativistic effects, so they remain to be normal semiconductors. Surprisingly, we find that the strong admixture of cation $p$ valence state and anion $p$ conduction state in rhombohedral phase, which does not have inversion symmetry, leads to loss of band inversion and makes the low-temperature phase $a$-SnTe a normal semiconductor. This raises an interesting question about the validity of previous theoretical ${ }^{4}$ and experimental ${ }^{10-17}$ studies about the topological behaviour of SnTe, suggesting that more studies are needed to clarify this issue.

\section{RESULTS AND DISCUSSION}

Figure 1 shows the calculated band structures of GeTe, SnTe and $\mathrm{PbTe}$ in ideal RS structure. They share common features over a large energy range of $20 \mathrm{eV}$, despite some systematic changes from compound to compound arising from the chemical shift of cations, changes in lattice constant, as well as spin-orbit splitting. Specifically, in the displayed energy window, all the conduction and valence band levels at high symmetry $k$-points occur in the

\footnotetext{
${ }^{1}$ Department of Physics, State Key Laboratory for Silicon Materials, Zhejiang University, Hangzhou, China; ${ }^{2}$ State Key Laboratory of Superlattices and Microstructures, Institute of Semiconductors, Chinese Academy of Sciences, Beijing, China and ${ }^{3}$ National Renewable Energy Laboratory, Golden, CO, USA.

Correspondence: J-W Luo (jwluo@semi.ac.cn)

Received 24 April 2015; revised 10 August 2015; accepted 10 August 2015
} 

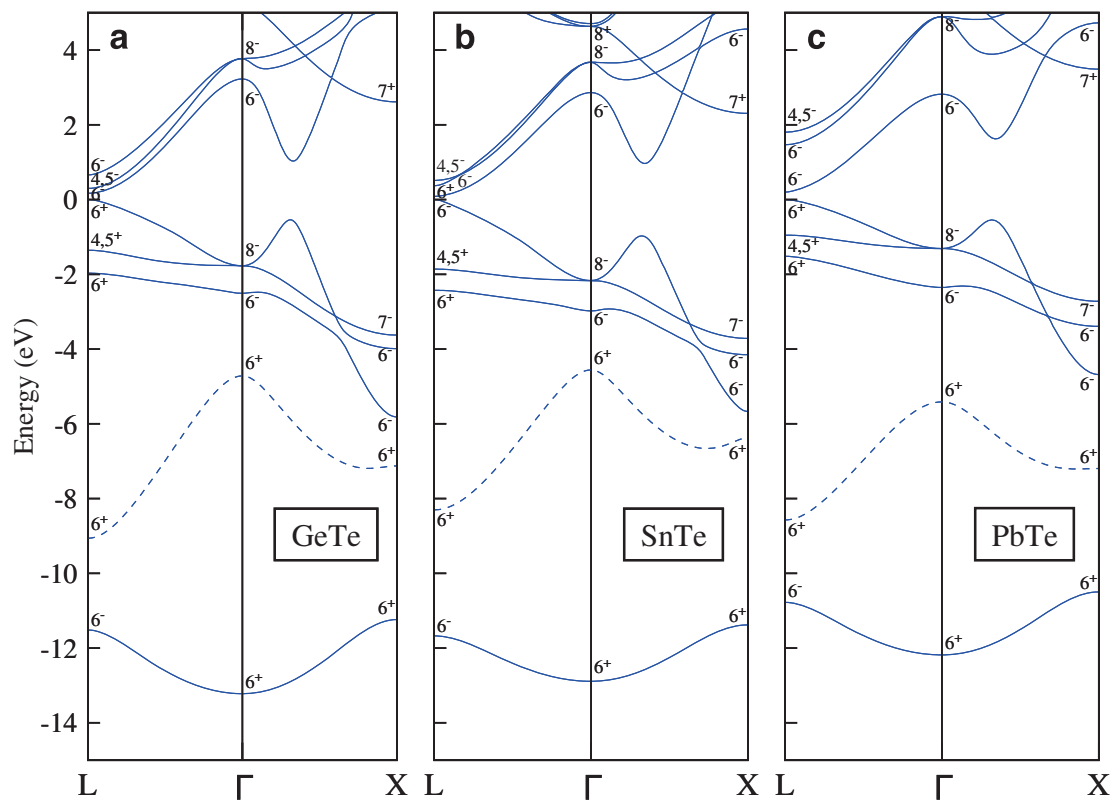

Figure 1. Calculated band structures of cubic (a) GeTe, (b) SnTe and (c) PbTe. The energy zero sets at the top of the valence band.

same order, except near the fundamental band gap, which is at the $L$ point. II-VI semiconductors (e.g., CdTe) are usually more stable in the zinc-blende structure and IV-VI semiconductors (e.g., $\mathrm{PbTe}$ ) are usually more stable in RS structure, although both have similar face-centred cubic BZ. Unlike conventional II-VI semiconductors where the cation $\mathrm{s}$ band becomes the lowest conduction band and is unoccupied, the large electronegativity of the groupIV elements results in their cation $s$ band (dashed lines in Figure 1) being occupied and below the anion $p$ band, forming the so-called lone-pair s band. Because of this, II-VI semiconductors usually have conduction band minimum (CBM) state occurring at $\Gamma$ point with mostly cation $s$ orbital character, whereas IV-VI semiconductors usually have CBM state at $L$ point with mostly cation $p$ orbital character. The $s-p$ coupling between cation $s$ band and anion valence $p$ band is forbidden at the high symmetry $\Gamma$ point but is allowed at the $L$ point. In IV-VI compounds, the occupied lone-pair $s$ band is low lying. The $s-p$ level repulsion pushes the valence $\mathrm{p}$ band up remarkably at $L$ point in energy, making it the valence band maximum (VBM) over the whole BZ. Thus, IV-VI semiconductors often have a direct band gap at the $L$ point despite the fact that all II-VI compounds have direct band gap at the $\Gamma$ point. ${ }^{18}$ In the following, we will discuss in more detail the band structures and chemical trends of GeTe, SnTe and $\mathrm{PbTe}$.

Figure 2 shows the band structures of RS SnTe calculated with and without SOC. We first examine the effects of the lone-pair $s$ band on the band structure of IV-VI semiconductors by neglecting the SOC, as shown in Figure 2a. At $\Gamma$ point, the anion $\mathrm{p}$ orbital valence band belongs to the triply degenerate $\Gamma_{15}$ irreducible representation (single group notation ${ }^{19}$ and chosen the cation sites as origin) but the low-lying cation lone-pair $\mathrm{s}$ band belongs to the $\Gamma_{1}$ irreducible representation, thus their coupling is strictly forbidden by symmetry since only states with the same symmetry can couple to each other. ${ }^{20}$ But the valence band $\Gamma_{15}$ state can couple with the high-lying cation $p$ derived conduction band $\Gamma_{15}$ state, pushing the valence band state down and the conduction band state up in energy. At $X$ point, the lone-pair s band has $X_{1}$ symmetry, whereas the three-fold degenerate valence $p$ orbital bands split into two bands with non-degenerate $X_{4}^{\prime}$ symmetry and doubly degenerate $X_{5}^{\prime}$ symmetry, respectively, and thus their coupling to the lone-pair band is also forbidden. Like at $\Gamma$ point, the $p-p$ coupling pushes the valence band ( $X_{5}^{\prime}$ state) down and the conduction band ( $X_{5}^{\prime}$ state) up in energy. Therefore, at both $\Gamma$ and $X$ points the low-lying lone-pair $s$ band could not directly influence the valence $p$ band, but the $p-p$ coupling leads to a level repulsion that lowers the valence $p$ band and raises the conduction $\mathrm{p}$ band. However, at the $L$ point, the valence anion p orbital bands split into a doubly degenerate $L_{3}$ state and a nondegenerate $L_{1}$ state, and the lone-pair $s$ band also has the $L_{1}$ symmetry, leading to inter-band coupling between $\mathrm{p}$ orbital $L_{1}$ state and lone-pair $s$ orbital $L_{1}$ state and resulting in a strong level repulsion. The emergent $s-p$ coupling at $L$ point significantly pushes the $L_{1}$ branch of the valence $\mathrm{p}$ states upward to a much higher energy than ones at $\Gamma$ point, making it the VBM. On the other hand, the $p-p$ coupling, which is allowed at $\Gamma$ and $X$ points, is forbidden by symmetry at $L$ point as shown in Figure $2 \mathrm{a}$, and thus the conduction band energy at the $L$ point is low. The conduction bands at $L$ point are further pushed down by high-lying conduction bands (with anion d character) of the same symmetry, making it the CBM. The SOC lowers the crystal symmetry and further lifts the degeneracy of $p$ orbital bands, as shown in Figure $2 \mathrm{~b}$, and may introduce additional inter-band coupling. However, the SOC introduced inter-band coupling is usually weak compared with the crystal field induced coupling unless the coupled states are nearly degenerate. Therefore, the general band structure is unaffacted by including SOC. This explains why the IV-VI RS compounds have a direct band gap occurs at the $L$ point.

Note that for conventional II-VI semiconductors in zinc-blende structure, the cation $s$ state is unoccupied and forms the conduction band. The lowest conduction $s$ band has different symmetry at $\Gamma$ point but same symmetry at $X$ and $L$ points as the valence $p$ derived states. The allowed $s-p$ coupling between conduction band and valence band at $X$ and $L$ points repels substantially the conduction band upwards and valence band downwards. This s-p coupling thus makes most of the zinc-blende II-VI semiconductors direct bandgap, having the CBM at the $\Gamma$ point and the VBM at the $\Gamma$ point.

We now turn to the abnormal trend of band ordering of the fundamental band gap among group-IV tellurides. Figure 1 shows that for GeTe and PbTe, the ordering of the bands at $L$ point is normal in the sense that they have a cation $p$ derived $L_{6}^{-}$(double group notation) CBM state and an anion $\mathrm{p}$ derived $L_{6}^{+}$VBM state. 

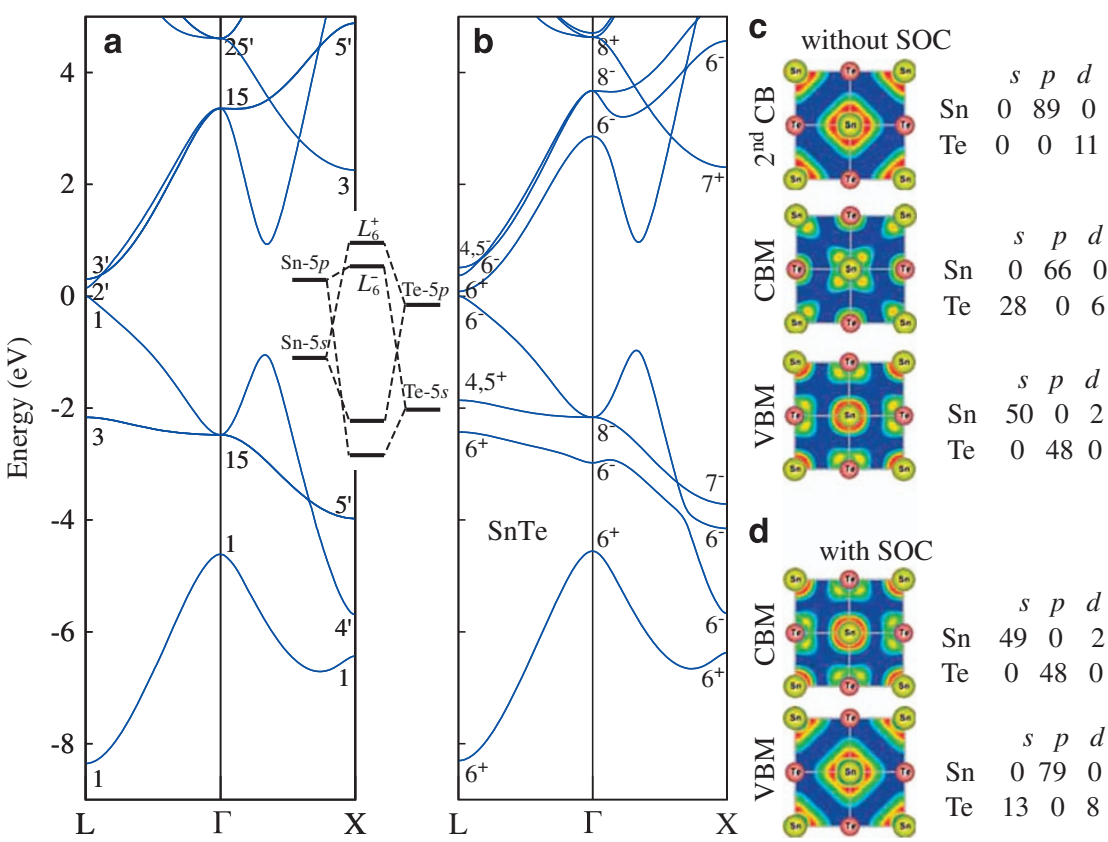

Figure 2. Calculated electronic band structures of SnTe (a) without SOC and (b) with SOC. The wave function squares as well as atomic orbital components (in percentage) of the CBM and VBM states are given in $\mathbf{c}$ and $\mathbf{d}$ for calculation without SOC and with SOC, respectively. Inset shows the schematic plot of s-p coupling in SnTe.

Table 1. Calculated band gaps at $L\left[E\left(L_{6}^{-}\right)-E\left(L_{6}^{+}\right)\right]$and $\Gamma\left[E\left(\Gamma_{6}^{-}\right)-E\left(\Gamma_{8}^{-}\right)\right]$points for GeTe, SnTe and PbTe at the experimental lattice constants $a$

\begin{tabular}{|c|c|c|c|c|c|c|c|}
\hline & \multirow[t]{2}{*}{ a $(\AA)$} & \multicolumn{2}{|c|}{$\mathrm{E}_{\mathrm{g}}(\mathrm{L})(\mathrm{eV})$} & \multirow[t]{2}{*}{$\mathrm{E}_{\mathrm{g}}(\Gamma)(\mathrm{eV})$} & \multicolumn{2}{|c|}{$\mathrm{E}_{\text {cation }}(\mathrm{eV})$} & \multirow[t]{2}{*}{$r_{\text {cation }}(\AA)$} \\
\hline & & Calc. & Expt. & & $E(s)$ & $\mathrm{E}(p)$ & \\
\hline GeTe & 5.996 (ref. 21) & 0.17 & 0.2 (ref. 21) & 4.99 & -11.93 & -4.05 & 1.52 \\
\hline SnTe & 6.300 (ref. 4) & -0.11 & -0.185 (ref. 4) & 5.05 & -10.78 & -3.87 & 1.72 \\
\hline $\mathrm{PbTe}$ & 6.428 (ref. 22) & 0.20 & 0.19 (ref. 22) & 4.13 & -12.04 & -3.50 & 1.81 \\
\hline
\end{tabular}

The calculated band gaps are compared with available experimental data. Calculated $\mathrm{s}$ and $\mathrm{p}$ valence atomic orbital energies (in eV) of $\mathrm{Ge}, \mathrm{Sn}$ and $\mathrm{Pb}$ are listed. The last column gives the atomic radii (in $\AA$ ) for these atoms.

However, for SnTe this band order is reversed, known as the Dimmock reversal, ${ }^{21}$ despite the fact that $\mathrm{Sn}$ element is in the middle of $\mathrm{Ge}$ and $\mathrm{Pb}$ in the group 14 column of the periodic table. Specifically, the CBM is a $L_{6}^{+}$state and the VBM is a $L_{6}^{-}$state, as shown in Figures 1 and $2 \mathrm{~b}$. It is usually expected that physical properties will change monotonically as the atomic number of the isovalent elements changes. For example, the band gaps of $\mathrm{ZnTe}$, $\mathrm{CdTe}$ and $\mathrm{HgTe}$ are reduced from 2.4 to $1.5 \mathrm{eV}$ and to $-0.3 \mathrm{eV} .^{18}$ Table 1 shows the calculated band gaps of GeTe, SnTe and PbTe, which are in good agreement with experimental results. Such band reversal can also be illustrated by examining the charge density distribution and the atomic orbital characters ${ }^{22}$ of the VBM and CBM states. Figure 3 shows clearly a switch of wavefunctions between CBM and VBM states from GeTe to SnTe and a switch back from SnTe to PbTe. This switch back indicates that the band gap order of SnTe is opposite to both of its lighter (GeTe) and heavier $(\mathrm{PbTe})$ isovalent neighbours. By projecting the CBM and VBM wavefunctions onto the atomic orbitals, we find in Figure 3 that the CBM of GeTe and PbTe and the VBM of SnTe are cation $p$ predominated with minor contributions from anion $\mathrm{s}$ and anion $\mathrm{d}$ orbitals, whereas the VBM of GeTe and PbTe and the CBM of SnTe are mainly from anion $\mathrm{p}$ with remarkable contributions from cation $\mathrm{s}$. This is consistent with the wave function distribution of CBM and VBM among GeTe, SnTe and PbTe. Therefore, the CBM symmetry of both GeTe and PbTe is $L_{6}^{-}$and their VBM symmetry is $L_{6}^{+}$. On the other hand, the order is reversed for the SnTe, i.e., the CBM is $L_{6}^{+}$and VBM is $L_{6}^{-}$. Because of the band inversion, SnTe was recently found to be a $\mathrm{TCl}$, but $\mathrm{GeTe}$ and $\mathrm{PbTe}$ are normal semiconductors.

The anomalous band gap ordering among GeTe, SnTe and PbTe can be explained as follows. Although the $\mathrm{Sn}$ element sits between $\mathrm{Ge}$ and $\mathrm{Pb}$ elements in the group 14 column of the periodic table, the energy of $\mathrm{Sn} 5 \mathrm{~s}$ orbital is 1.15 and $1.26 \mathrm{eV}$ higher than the $\mathrm{Ge} 4 \mathrm{~s}$ and $\mathrm{Pb} 6 \mathrm{~s}$ orbitals, respectively, and thus more delocalised in real space and closer in energy to the highlying Te $4 p$ orbital (Table 1). The abnormally low Ge 4 s orbital in energy is due to the incomplete screening of the nucleus by the $3 \mathrm{~d}$ orbitals, whereas, the low $\mathrm{Pb} 6 \mathrm{~s}$ orbital energy is due to the large relativistic effect of $\mathrm{Pb}^{23}$ The high energy and delocalised character of Sn 5s orbital lead to stronger s-p coupling in SnTe than in other two compounds. In fact, the s-p coupling (Figure 2a inset) in SnTe is so strong that the $L_{6}^{+}$(Figures $1 \mathrm{~b}$ and $2 \mathrm{~b}$ ) state is pushed up even above the $L_{6}^{-}$state and leads to the band inversion. Such $s-p$ coupling also appears in the other two compounds, but it is not strong enough to leading to band inversion in GeTe and PbTe.

Besides the cation $s$ atomic energy levels, there are several other factors that also have consequences for the band ordering. First, the cation atomic radius increases in the order $r(\mathrm{Ge})<r(\mathrm{Sn})$ $<r(\mathrm{~Pb})$ (Table 1) so are the bond lengths in the IV-Te compounds. 


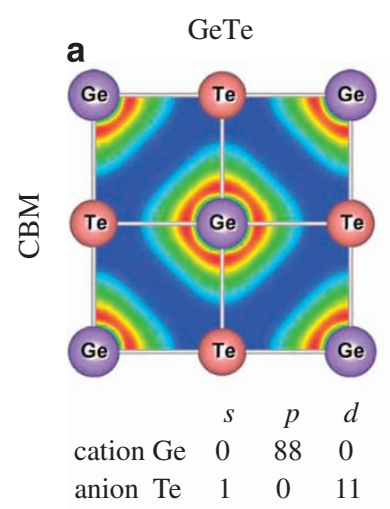

$$
\text { d }
$$

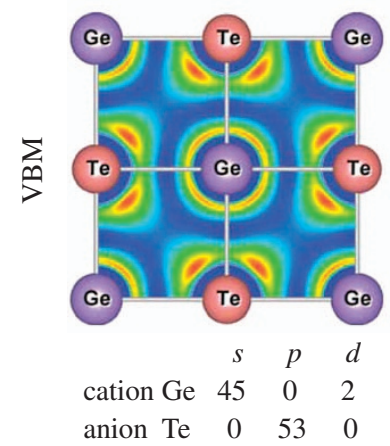

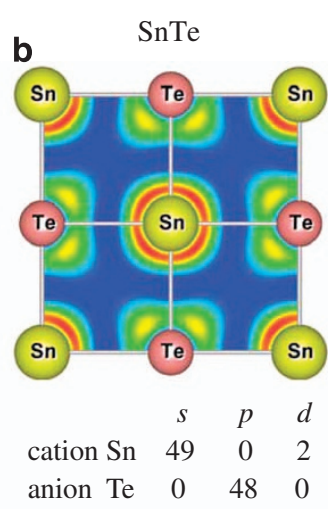

e

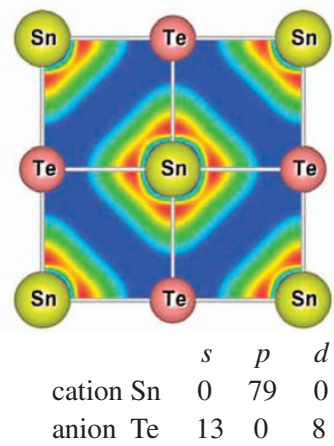

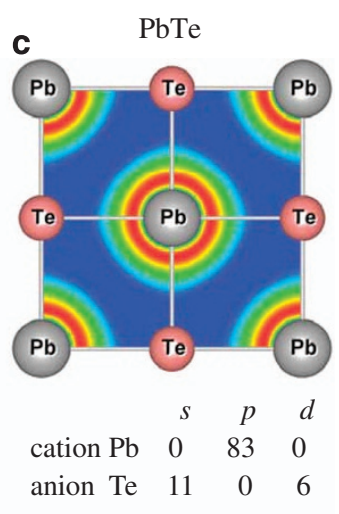

f

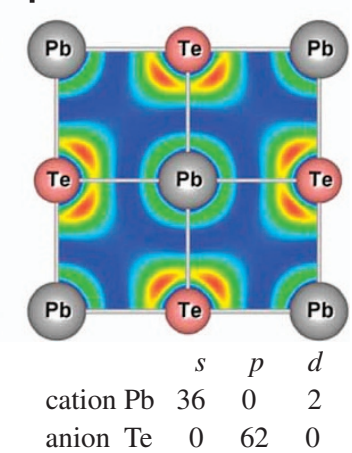

Figure 3. Wave function square (or charge distribution) of CBM and VBM states that occur at the $L$ point for cubic (a and d) GeTe, (b and e) $\mathrm{SnTe}$ and (c and f) PbTe. The site and angular momentum projected wave function characters within the projector augmented wave spheres of the CBM and VBM states in percentage are listed for each state.

The shorter the bond length is, the larger the $s-p$ repulsion could be. Among the three compounds, GeTe has the shortest bond length, so this factor will favour GeTe to have inverted band gap. Second, SOC is generally regarded as the main driving force to invert band order for three-dimensional $\mathrm{Tls}^{2}{ }^{2}$ The $\mathrm{SOC}$ in $\mathrm{Pb}$ is the largest among the three cation elements as evidenced by the fact that the atomic p orbital spin-orbit splitting is $0.29,0.71$ and $2.4 \mathrm{eV}$ for $\mathrm{Ge}, \mathrm{Sn}$ and $\mathrm{Pb}^{24}$ respectively, and the spin-orbit splitting of the conduction band at the $\Gamma$ point $\left[E\left(\Gamma_{8}^{-}\right)-E\left(\Gamma_{6}^{-}\right)\right]$is $0.55,0.81$ and $2.1 \mathrm{eV}$ in GeTe, SnTe and PbTe, respectively, which are in good agreement with the atomic trend. The spin-orbit splitting pushes the CBM down and VBM up and therefore will favour $\mathrm{PbTe}$ to having band inversion. Furthermore, the Ge $4 \mathrm{p}$ is 0.18 and $0.55 \mathrm{eV}$ lower in energy than $\mathrm{Sn} 5 \mathrm{p}$ and $\mathrm{Pb} 6 \mathrm{p}$ orbitals, respectively. The lower energy of the cation $p$ orbital also favors the band inversion. The net effect of the 'balancing acts' is that all the three IV-Te compounds have similar band structures with SnTe being band inverted.

It is interesting to see that the band gaps at $\Gamma$ point $E_{g}\left(\Gamma_{6}^{-}-\Gamma_{8}^{-}\right)$ also show non-monotonic behaviour of the three compounds, but in the exactly opposite way, that is, $E_{g}\left(\Gamma_{6}^{-}-\Gamma_{8}^{-}\right)$decreases from $\mathrm{SnTe}$ to GeTe to PbTe (Table 1). From Figure 1, it can be seen that $E_{g}\left(\Gamma_{6}^{-}-\Gamma_{8}^{-}\right)$is between the bonding and anti-bonding states of anion $\mathrm{p}$ and cation $\mathrm{p}$ orbitals. SnTe has the largest $E_{g}\left(\Gamma_{6}^{-}-\Gamma_{8}^{-}\right)$ because Sn $5 p$ orbital has higher energy than that of Ge $4 p$ orbital energy. For $\mathrm{PbTe}$, although $\mathrm{Pb}$ has even higher $6 \mathrm{p}$ orbital energy than $\mathrm{Sn} 5 \mathrm{p}$, the larger atomic size and strong $\mathrm{SOC}$ of $\mathrm{Pb}$ reduces $E_{g}\left(\Gamma_{6}^{-}-\Gamma_{8}^{-}\right)$of PbTe.

For GeTe and SnTe, the stable structure below the transition temperature $T_{c}$ is actually the rhombohedral distorted $a$ phase. The $T_{\mathrm{c}}$ is $670 \mathrm{~K}$ and $98 \mathrm{~K}$, respectively, for GeTe and $\mathrm{SnTe}^{25}$ in the low carrier concentration limit. For PbTe, the ferroelectric phase transition does not occur at low temperature. ${ }^{26}$ The lattice parameters of the primitive rhombohedral cell ${ }^{27-30}$ are $a_{r}=4.293$
$\AA, a_{r}=58.032^{\circ}$ for GeTe and $a_{r}=4.475 \AA, a_{r}=59.879^{\circ}$ for SnTe. The RS-to-rhombohedral ferroelectric phase transition is accompanied by a relative shift $\tau(0.028$ for GeTe and 0.014 for $\mathrm{SnTe}$ ) of the cation and anion sublattices along the cubic $<111>$ direction that breaks the space inversion symmetry. The four degenerate $L$ points in RS BZ split into three $L$ points with symmetry $C_{s}$ and one $Z$ point with symmetry $C_{3 v}$ in the rhombohedral BZ.

The calculated direct energy band gap at $L$ point is $0.59 \mathrm{eV}$ for $a$-GeTe (rhombohedral structure), much larger than $0.17 \mathrm{eV}$ for $\beta$-GeTe (cubic RS structure) due to atom displacement in rhombohedral structure, which lowers the crystal symmetry by lifting the inversion symmetry and leads to reduced $s-p$ coupling and admixture between the cation $p$ valence state and anion $p$ conduction state at $L$ point, hence opens up a large gap between valence and conduction states. For $a-S n T e$, the reduced $s-p$ coupling and strong admixture between cation $\mathrm{p}$ valence state and anion $p$ conduction state leads to loss of band inversion and makes the material a normal semiconductor with a band gap of $0.20 \mathrm{eV}$ (Figure 4). It is interesting to notice that if we assume there is no atom displacement in $a$-SnTe (i.e., $\tau=0$ ), the character of VBM and CBM is same as in the $\beta$-SnTe and the band gap is still inverted.

The topological phase transition induced by the structure phase transition in SnTe is consistent with theoretical results in ref. 28 . It is believed that the $T_{\mathrm{c}}$ depends strongly on the carrier concentration and the $T_{c}$ can be much lower when there is high carrier concentration. ${ }^{31}$ Although some of the reported experiments $^{10-17}$ were conducted at a temperature below the low carrier concentration $T_{c}$ limit, topological phase transition induced by structure phase transition have not been observed to our knowledge. Further experimental confirmation is called for to test our prediction that there is a topological phase transition between RS $\beta$-SnTe and rhombohedral $a$-SnTe. 

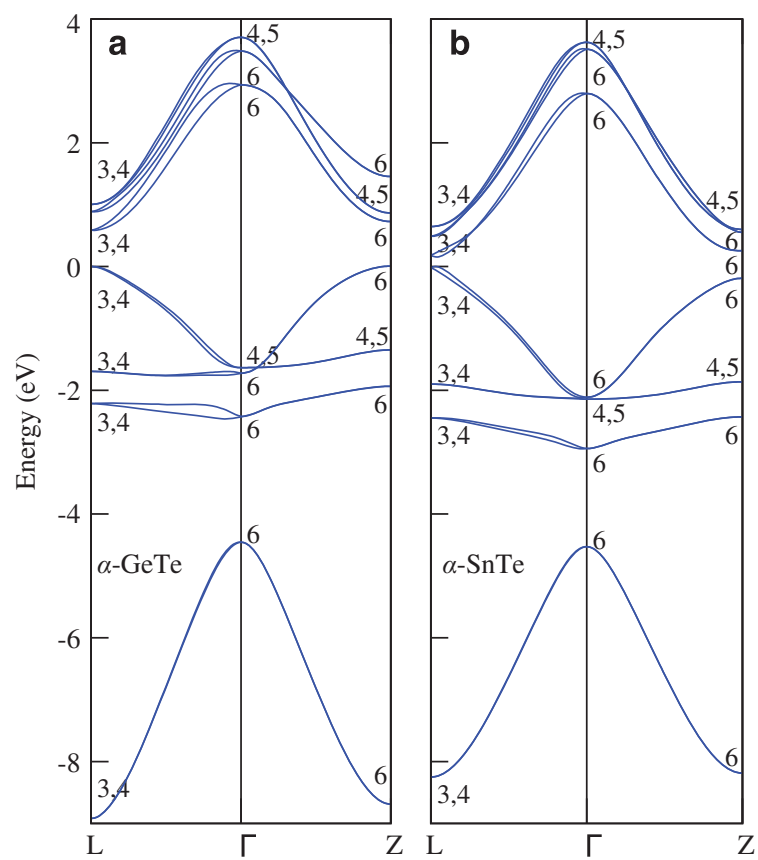

Figure 4. Calculated energy band structure of (a) GeTe and (b) SnTe in rhombohedral structure.

\section{Conclusions}

We have investigated the electronic structure of group-IV tellurides GeTe, SnTe and PbTe using first-principles calculation. The abnormal trend in their electronic band structures, i.e., a band inversion in SnTe but not in GeTe and $\mathrm{PbTe}$, is explained by symmetry analysis and the atomic energy levels and sizes. We show that the low-lying cation $s$ lone-pair band state couples strongly with the high-lying anion valence $\mathrm{p}$ band states at $L$ point and this $s-p$ coupling pushes the anion valence $p$ band up to become the VBM. The Ge 4 s orbital energy is lower than Sn 5s orbital energy due to the incomplete screening of the nucleus by the $3 \mathrm{~d}$ orbitals. The $\mathrm{Pb} 6 \mathrm{~s}$ orbital energy is lower than $\mathrm{Sn} 5 \mathrm{~s}$ orbital energy due to the large relativistic effect of $\mathrm{Pb}$ atom. The highest cation $s$ orbital energy of $\mathrm{Sn}$ among the three cation atoms, leading to the largest $s-p$ coupling in SnTe with respect to GeTe and $\mathrm{PbTe}$, makes $\mathrm{SnTe}$ band gap (at the $L$ point) inverted, thus explains the peculiar chemical trends in the band structure among the three compounds. We also demonstrate that internal atom displacement in $a$-GeTe and $a$-SnTe induces strong admixture between CBM and VBM and reduces s-p coupling, thus increases the band gap. The $a$-SnTe is found to be a normal semiconductor because of reduced $s-p$ coupling and admixture of $p$ orbitals, which requires further experimental verification. Our study illustrates that besides reported $\mathrm{SOC}^{2}{ }^{2}$ strain, ${ }^{2}$ alloying ${ }^{32}$ and interface dipole field, ${ }^{33}$ inter-level coupling can also be an effective pathway to design Tls. Specifically, we could choose different atoms with different atomic orbital energies, apply pressure or strain to change the atom-atom bond length, regulate the charge state (Coulomb $\mathrm{U}$ ) of an atom to control inter-level coupling.

\section{COMPUTATIONAL METHODS}

The electronic structure and total energy are calculated using first-principles density functional theory as implemented in the Vienna $\mathrm{Ab}$ initio simulation package. ${ }^{34}$ The local density approximation ${ }^{35,36}$ is used to deal the correlation effects whereas the modified Becke and Johnson exchange potential (termed TB-mBJ) ${ }^{37}$ is adopted to improve the local density approximation description of band structures. The projector augmented wave method ${ }^{38,39}$ is used to treat the interaction between the valence electrons and the core electrons. ${ }^{39}$ The valence configurations of $\mathrm{Ge}, \mathrm{Sn}, \mathrm{Pb}$ and Te atoms considered in the calculations are $3 \mathrm{~d}^{10} 4 \mathrm{~s}^{2} 4 \mathrm{p}^{2}$, $4 d^{10} 5 s^{2} 5 p^{2}, 5 d^{10} 6 s^{2} 6 p^{2}$ and $5 s^{2} 5 p^{4}$, respectively. A plane-wave expansion up to $400 \mathrm{eV}$ is applied and a $\Gamma$-centred $8 \times 8 \times 8$ Monkhorst-Pack ${ }^{40} k$-mesh is used for the Brillouin zone (BZ) integration. Spin-orbit interaction, implemented in the Vienna Ab initio simulation package code following the approach of Kleinman and Bylander, ${ }^{41}$ is included in the self-consistent calculations because it involves heavy elements of $\mathrm{Ge}, \mathrm{Sn}, \mathrm{Pb}$ and Te and has an important role in the topological behaviour of the compounds. The experimental lattice constants (Table 1) are used in TB-mBJ band structure calculation as suggested in ref. 42. to avoid uncertainty in the calculated band structure due to a small error in calculated lattice constants. ${ }^{4}$ It yields band gaps of semiconductors with an accuracy comparable to GW methods but is computationally much less expensive. It is worth to note that the TB-mBJ approach remarkably improves over the original BJ approach for the description of band gaps ${ }^{37}$ and the local density approximation error for $p-p$ like band gap such as those in IV-Te compounds is expected to be small. ${ }^{6}$

\section{ACKNOWLEDGEMENTS}

This work was supported by Natural Science Foundation of China (No. 61290305, 11374259,11374293 and 61474116). JWL was supported by the National Young 1000 Talents Plan. The work at NREL was funded by the US Department of Energy, under Contract No. DE-AC36-08GO28308.

\section{COMPETING INTERESTS}

The authors declare no conflict of interest.

\section{REFERENCES}

1 Hasan, M. Z. \& Kane, C. L. Colloquium: topological insulators. Rev. Mod. Phys. 82, 3045-3067 (2010).

2 Qi, X.-L. \& Zhang, S.-C. Topological insulators and superconductors. Rev. Mod. Phys. 83, 1057-1110 (2011).

3 Fu, L. Topological crystalline insulators. Phys. Rev. Lett. 106, 106802 (2011).

$4 \mathrm{Hsieh}, \mathrm{T}$. H. et al. Topological crystalline insulators in the SnTe material class. Nat. Commun. 3, 982 (2012).

5 Springholz, G. in Molecular Beam Epitaxy: From Research to Mass Production (ed. Henini M.) (Elsevier, 2013).

6 Wei, S.-H. \& Zunger, A. Electronic and structural anomalies in lead chalcogenides. Phys. Rev. B 55, 13605-13610 (1997).

7 Jin, S., Cai, C., Bi, G., Zhang, B., Wu, H. \& Zhang, Y. Two-dimensional electron gas at the metastable twisted interfaces of $\mathrm{CdTe} / \mathrm{PbTe}(111)$ single heterojunctions. Phys. Rev. B 87, 235315 (2013).

8 Zhang, B. et al. Quantum oscillations in a two-dimensional electron gas at the rocksalt/zincblende interface of PbTe/CdTe (111) heterostructures. Nano Lett. 15, 4381-4386 (2015).

9 Zhang, B. et al. Phonon blocking by two dimensional electron gas in polar $\mathrm{CdTe} / \mathrm{PbTe}$ heterojunctions. Appl. Phys. Lett. 104, 161601 (2014).

10 Tanaka, Y. et al. Experimental realization of a topological crystalline insulator in SnTe. Nat. Phys. 8, 800-803 (2012).

11 Dziawa, P. et al. Topological crystalline insulator states in $\mathrm{Pb}_{1-x} \mathrm{Sn}_{x} \mathrm{Se}$. Nat. Mater. 11, 1023-1027 (2012).

$12 \mathrm{Xu}, \mathrm{S}$. Y. et al. Observation of a topological crystalline insulator phase and topological phase transition in $\mathrm{Pb}_{1-x} \mathrm{Sn}_{x}$ Te. Nat. Commun. 3, 1192 (2012).

13 Yan, C. et al. Experimental observation of dirac-like surface states and topological phase transition in $\mathrm{Pb}_{1-x} \mathrm{Sn}_{x} \mathrm{Te}$ (111) films. Phys. Rev. Lett. 112, 186801 (2014).

14 Okada, Y. et al. Observation of dirac node formation and mass acquisition in a topological crystalline insulator. Science 341, 1496-1499 (2013).

15 Zhang, D. et al. Quasiparticle scattering from topological crystalline insulator SnTe (001) surface states. Phys. Rev. B 89, 245445 (2014).

16 Assaf, B. A. et al. Quantum coherent transport in SnTe topological crystalline insulator thin films. Appl. Phys. Lett. 105, 102108 (2014).

17 Taskin, A. A., Yang, F., Sasaki, S., Segawa, K. \& Ando, Y. Topological surface transport in epitaxial $\mathrm{SnTe}$ thin films grown on $\mathrm{Bi}_{2} \mathrm{Te}_{3}$. Phys. Rev. B 89, 121302 (2014).

18 Madelung, O. Semiconductors: Data Handbook 3rd edn (Springer, 2004).

19 Koster, G. F., Dimmock, J. O., Wheeler, R. G. \& Statz, H. Properties of the Thirty-Two Point Groups (MIT Press, 1963). 
20 Harrison, W. A. Electronic Structure and the Properties of Solids: The Physics of the Chemical Bond (Dover, 1989).

21 Dimmock, J. O., Melngailis, I. \& Strauss, A. J. Band structure and laser action in $\mathrm{Pb}_{x} \mathrm{Sn}_{1-x}$ Te. Phys. Rev. Lett. 16, 1193-1196 (1966).

22 Dimmock, J. O. \& Wright, G. B. Band edge structure of PbS, PbSe, and PbTe. Phys. Rev. 135, A821-A830 (1964).

23 Phillips, J. C. Bonds and Bands in Semiconductors (Academic Press, 1973).

24 Herman, F., Kuglin, C. D., Cuff, K. F. \& Kortum, R. L. Relativistic corrections to the band structure of tetrahedrally bonded semiconductors. Phys. Rev. Lett. 11, 541-545 (1963).

25 Bussmann-Holder, A., Bilz, H. \& Vogl, R. in Dynamical Properties of IV-VI Compounds (Springer, 1983).

26 Bate, R. T., Carter, D. L. \& Wrobel, J. S. Paraelectric behavior of PbTe. Phys. Rev. Lett 25, 159-162 (1970).

27 Wdowik, U. D., Parlinski, K., Rols, S. \& Chatterji, T. Soft-phonon mediated structural phase transition in GeTe. Phys. Rev. B 89, 224306 (2014).

28 Plekhanov, E., Barone, P., Di Sante, D. \& Picozzi, S. Engineering relativistic effects in ferroelectric SnTe. Phys. Rev. B 90, 161108 (2014).

29 Goldak, J., Barrett, C. S., Innes, D. \& Youdelis, W. Structure of alpha GeTe. J. Chem. Phys. 44, 3323-3325 (1966)

30 Shaltaf, R., Durgun, E., Raty, J. Y., Ghosez, P. \& Gonze, X. Dynamical, dielectric, and elastic properties of GeTe investigated with first-principles density functional theory. Phys. Rev. B 78, 205203 (2008).

31 lizumi, M., Hamaguchi, Y., Komatsubara, F. K. \& Kato, Y. Phase transition in SnTe with low carrier concentration. J. Phys. Soc. Jpn. 38, 443-449 (1975).

32 Yan, B. \& Zhang, S.-C. Topological materials. Rep. Prog. Phys. 75, 096501 (2012).

33 Zhang, D., Lou, W., Miao, M., Zhang, S.-C. \& Chang, K. Interface-induced topological insulator transition in $\mathrm{GaAs} / \mathrm{Ge} / \mathrm{GaAs}$ quantum wells. Phys. Rev. Lett. 111 156402 (2013).
34 Kresse, G. \& Furthmüller, J. Efficiency off ab-initio total energy calculations for metals and semiconductors using a plane-wave basis set. Comput. Mat. Sci. 6 15-50 (1996)

35 Ceperley, D. M. Ground state of the electron gas by a stochastic method. Phys. Rev. Lett. 45, 566-569 (1980).

36 Perdew, J. P. \& Zunger, A. Self-interaction correction to density-functional approximations for many-electron systems. Phys. Rev. B 23, 5048-5079 (1981).

37 Tran, F. \& Blaha, P. Accurate band gaps of semiconductors and insulators with a semilocal exchange-correlation potential. Phys. Rev. Lett. 102, 226401 (2009).

38 Blöchl, P. E. Projector augmented-wave method. Phys. Rev. B 50, 17953-17979 (1994).

39 Kresse, G. \& Joubert, D. From ultrasoft pseudopotentials to the projector augmented-wave method. Phys. Rev. B 59, 1758-1775 (1999).

40 Monkhorst, H. J. \& Pack, J. D. Special points for Brillouin-zone integrations. Phys. Rev. B 13, 5188-5192 (1976).

41 Kleinman, L. \& Bylander, D. M. Efficacious form for model pseudopotentials. Phys. Rev. Lett. 48, 1425-1428 (1982).

42 Kim, Y.-S., Marsman, M., Kresse, G., Tran, F. \& Blaha, P. Towards efficient band structure and effective mass calculations for III-V direct band-gap semiconductors. Phys. Rev. B 82, 205-212 (2010).

This work is licensed under a Creative Commons Attribution 4.0 International License. The images or other third party material in this article are included in the article's Creative Commons license, unless indicated otherwise in the credit line; if the material is not included under the Creative Commons license, users will need to obtain permission from the license holder to reproduce the material. To view a copy of this license, visit http://creativecommons.org/licenses/ by/4.0/ 